\title{
Study on In-Ovo Vaccination by Needle-Free Injection
}

\author{
Ko-Jung Huang ${ }^{1}$, Wei-Chih Chen ${ }^{1}$, Ching-Wei Cheng ${ }^{2}$ \\ ${ }^{1}$ Department of Bio-industrial Mechatronics Engineering, National Chung Hsing University \\ No.145 Xingda Rd., South Dist., Taichung City 402, Taiwan (R.O.C.) \\ First. g8925008@gmail.com \\ ${ }^{2}$ College of Intelligence, National Taichung University of Science and Technology \\ No.129, Sec.3, Sanmin Rd, North Dist., Taichung City 404, Taiwan (R.O.C.) \\ Third.cwcheng@nutc.edu.tw
}

\section{Extended Abstract}

Chick immunization in Taiwan in the current stage is done in the traditional artificial way via chick subcutaneous injection, eye drops, spray vaccine, or getting the vaccine mixed with feed and other methods for epidemic prevention. In addition to the traditional artificial way, in 1982, Sharma and Burmester proved the success of egg injection via Marek's vaccine immunization under normal laboratory conditions for the first time [1]. Eighty-five percent of the US chicken industry deployed egg injection as an important method of chicken embryo immunization.

Presently, many countries have developed the automated egg syringe system. Due to congenital biological differences of individual eggs, the vaccine cannot be accurately injected; there is lack of control of the needle-hit position. This may affect the growth of the chicken embryo.

Wakenell et al. Published the Marek's vaccine in 2002 and the efficacy of the vaccine. Sparring time at embryonic age of 18 days, vaccines in the amniotic cavity can be absorbed with other nutrients together with chicken embryo. If the chick embryo age is more than 19 days, the vaccination position in the chicken embryo body itself is of a better condition. In general, the embryo inoculation is to inject the vaccine into the amniotic cavity or directly into the chicken embryo. Its immune affects than the traditional subcutaneous injection more effective. [2]

The purpose of this research is to develop a needle-free syringe. The following experiment is divided into two processes and it could confirm the feasibility of needleless injection for in-ovo vaccination, one is to use different force tests on hatching egg embryos with a needleless pneumatic syringe, and to inject the egg embryos with the optimal force. The other is that chicken embryo injected with saline and IB MA5 vaccination at 18.5 days with the optimal force. The results shown that there was not much difference between the physiological status and hatchability of the chickens in the control group and the experimental group. Furthermore, blood samples used to estimate the antibody titres once a week for 3 times after incubation, and most chicken could produce antibodies themselves.

The suitable injection pressure is at the pressure of 3.44 bar and 3.79 bar, which can make the injection into the amniotic cavity. The needle injection was carried out with the appropriate injection force, there was no significant effect on the hatching rate. From the results of IB vaccination and blood test, it showed that chicks would have a more pronounced titre in 2 or 3 weeks after hatching. Titre of chicks in third weeks distributed among 165 to 4636 . Therefore, it is possible to carry out an in ovo vaccination with a needle-free injection device at a suitable injection force.

Those results help to develop automated equipment for in-ovo vaccine injection, effectively improve operating efficiency and hygiene of vaccination operations, establish a safe environment for poultry industry, and solve problems such as the non-implemented vaccination of birds with artificial IB vaccines.

\section{References}

[1] Koprowski, H. and C. H. Cox. 1948. Studies on chick-embryo-adapted rabies virus; culture characteristics and pathogenicity. J. Immunol. Vol. 60, no. 4, pp. 533-544.

[2] Wakenell, P. S., T. Bryan, J. Schaeffer, A. Avakian, C. Williams, C. Whitfill. 2002. Effect of in ovo vaccine delivery route on herpesvirus of turkeys/SB-1 efficacy and viremia. Avian Dis. Vol. 46, no. 2, pp. 274-280. 
[3] Chen, D., R. L. Endres, C. A. Erickson, K. F. Weis, M. W. McGregor, Y. Kawaoka, L. G. Payne. 2000. Epidermal immunization by a needle-free powder delivery technology : immunogenicity of influenza vaccine and protection in mice. Nat Med. Vol. 6, no. 10, pp. 1187-1190.

[4] Williams, C. J. and B. A. Hopkins. 2011. Field evaluation of the accuracy of vaccine deposition by two different commercially available in ovo injection systems. Poult. Sci. vol. 90, no. 1, pp. 223-226.

[5] Ziegler, A. 2007. Needle-free injection--science fiction or comeback of an almost forgotten drug delivery system. Med Monatsschr Pharm. Vol. 30, no. 8, pp. 297-303. 